\title{
Diagnostic Dilemma of Polymorphous Low Grade Adenocaicinoma of Hard Palate
}

Seema Monga, Junaid Nasim Malik, Arun Parkash Sharma, Sujata Jetley

Department of Otorhinolaryngology and Head \& Neck Surgery, HIMSR \& HAHC

Hospital, Jamia Hamdard University, Delhi-1 10062, India.

Abstract:

Polymorphous low grade adenocarcinoma (PLGA) is an indolent malignant tumor affecting the minor salivary gland almost exclusively. It is important to differentiate PLGA from other salivary gland tumors with myoepithelial differentiation such as pleomorphic adenoma and adenoid cystic carcinoma. We report a case of a 55 year old male who presented with an asymptomatic swelling on the hard palate for the past one year. FNAC of the swelling was suggestive of pleomorphic adenoma and surgical excision with adequate margins was done. Histopathological and immunohistochemistry examination of the excised specimen came out to be PLGA. A very inquisitive work up including adequate sized biopsy and immunohistochemistry is recommended for the diagnosis of PLGA. The clinician and the cytopathologist should be aware of the characteristic cytomorphologic and immunohistochemistry features of PLGA to distinguish it from pleomorphic adenoma as happened in the present case.

Key words: Salivary Gland Neoplasms, Adenoma, Pleomorphic, Adenoid Cystic Carcinoma, Immunohistochemistry, Hard Palate.

\section{Introduction}

Polymorphous low grade adenocarcinoma (PLGA) is a malignant epithelial tumor of salivary gland characterized by cytological uniformity, morphological diversity, an infiltrative growth pattern and low metastatic potential [1]. It is important and exigent to differentiate it from other salivary gland tumors with myoepithelial differentiation such as pleomorphic adenoma (PA), adenoid cystic carcinoma (ACC) and epithelial-myoepithelial carcinoma, thus a diagnostic challenge $[2,3]$. PLGA is the most second common primary minor salivary gland malignancy after mucoepidermoid carcinoma comprising $9-26 \%$ of all salivary gland malignancy, the age of presentation being 30-70 years with female ratio twice the male $[3,4]$.

It is most commonly seen in the palate (49-77.8\%), followed by either buccal mucosa or the upper lip (7.4-13.4\%) and can also involve the floor of mouth, lower lip, alveolar ridge and tongue [4,5]. Clinically it presents as a firm, well circumscribed, painless, slow growing mass mostly covered by nonulcerated mucosa resembling a benign neoplasm which can be responsible for the delay in diagnosis

Corresponding Author: Dr. Seema Monga

Email: dr.seema.monga@gmail.com

Received: December 28, 2013 | Accepted: February 10, 2014 | Published Online: March 10, 2014 This is an Open Access article distributed under the terms of the Creative Commons Attribution License (creativecommons.org/licenses/by/3.0)

Conflict of interest: None declared | Source of funding: Nil | DOl: http://dx.doi.org/10.17659/01.2014.0023 
for years. The lesion may be fixed to underlying structures eroding \& infiltrating the underlying bone and even present with perivascular and perineural invasion. We report a case of a 55 year old man with asymptomatic swelling on hard palate whose clinical behavior and FNAC picture was suggestive of PA. It was surgically excised and histopathology was suggestive of PLGA.

\section{Case Report}

A 55 year old male presented with nearly one year history of a painless swelling on his hard palate without any associated history of ulceration, discharge or bleeding from the swelling or difficulty in swallowing. He had no complaints of weight loss, nasal obstruction or nasal discharge. He was nonsmoker with history of occasional alcohol intake.

A midline $1.5 \times 2 \mathrm{~cm}$ mass was present on the hard palate covered with normal mucosa. It was firm, well circumscribed and non-tender [Fig.1 a]. FNAC of the swelling was done which showed features of pleomorphic adenoma. CECT scan of the head \& neck showed the tumor without any local or bone infiltration and no neck lymphadenopathy. In view of the benign nature of the disease surgical excision with adequate safety margins was performed [Fig. 2]. Although the underlying bone was intact and not involved it was superficially drilled and the base cauterized [Fig. 1b]. As the wound was small it was

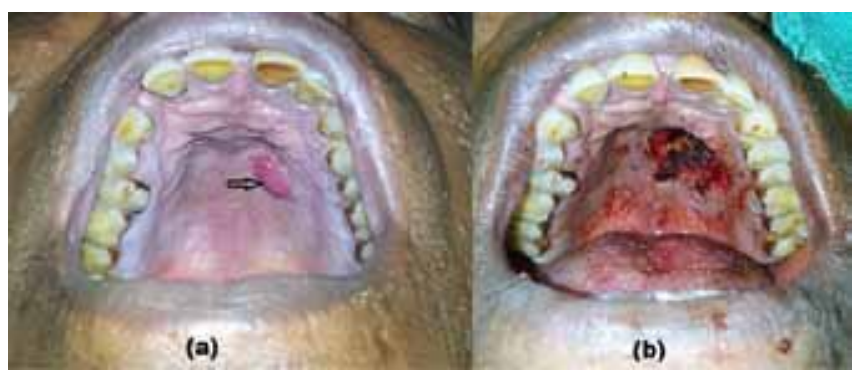

Fig. 1: (a) Clinical picture of PLGA hard palate, (b) site after excision. allowed to heal by secondary intention and patient was advised oral hygiene measures and a short course of antibiotics. Histopathological examination showed a low-grade tumor with tubular, papillary and papillary-cystic areas. One single cell type was seen forming these structures with round pale nuclei, fine evenly distributed chromatin, indistinct nucleoli and no mitotic activity. Necrosis was absent and the stroma was eosinophilic and hyalinized in nature [Fig.3a]. A histological diagnosis of polymorphous low grade adenocarcinoma was made. Immunohistochemical staining was done and showed positivity for S-100 protein and Vimentin, which further supported this histological diagnosis [Fig.3b].

As the disease was removed with clear margins the patient was not started on radiotherapy but kept on initial weekly follow up. After three months, patient presented with granulations on the healing wound edges. In view of significant chances of recurrence, biopsy of the granulations was done and sent for HPE which was suggestive of inflammatory infiltrate and no evidence of malignancy was seen. After

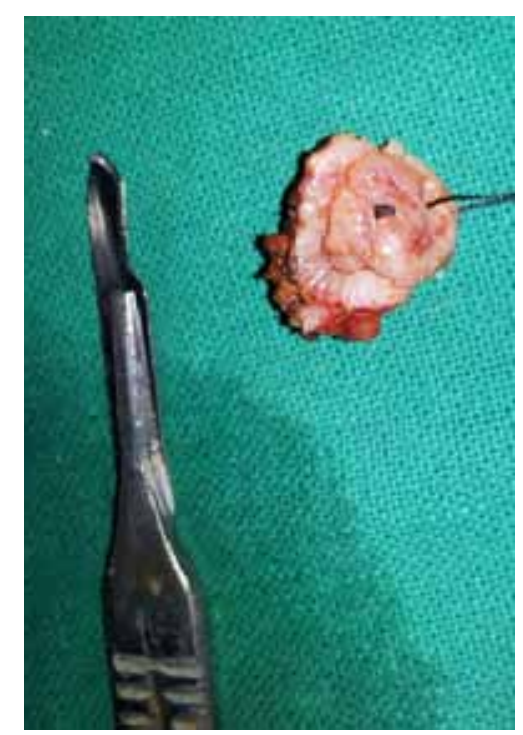

Fig.2: Excised PLGA tumor with adequate margins. 
close follow up of six months the patient remains disease free.

\section{Discussion}

Polymorphous low grade adenocarcinoma (PLGA) has a different clinical pattern from other malignant salivary gland neoplasms as it is characterized by its absence of symptoms, slow rate of growth, minimal aggressiveness and favorable prognosis with low metastatic potential [6]. This case evidences low grade of malignancy and slow rate of growth of PLGA which is demonstrated in the fact that the patient was aware of slow growing mass in his mouth for a year but didn't seek medical attention due to no symptoms.

Amongst the neoplasms of minor salivary glands, PLGA is the second most common malignant whereas PA is the most commonly benign tumor. PLGA is usually misdiagnosed with pleomorphic adenoma (PA) as happened in the present case. Both tumors have a similar clinical behavior and differentiating them histopathologically may be difficult due to presence of infiltrative margins where the capsule is not complete. Also presence of hyaline in the stroma in a limited PLGA specimen may be mistaken for the mucomyxoid matrix of PA [6].

Among the malignant neoplasms of minor salivary glands, PLGA is occasionally confused with adenoid cystic carcinoma (ACC) due to overlapping histological features. ACC being aggressive, having faster growth rate and higher rate of distant metastasis requires a more radical surgical approach often combined with adjuvant radiotherapy stressing the need to precisely differentiate it from PLGA [7].

Accuracy of histopathological diagnosis depends upon sufficient size of specimen to reveal heterogenous histologic spectrum, infiltrative nature and perineural invasion typical of PLGA. A solitary

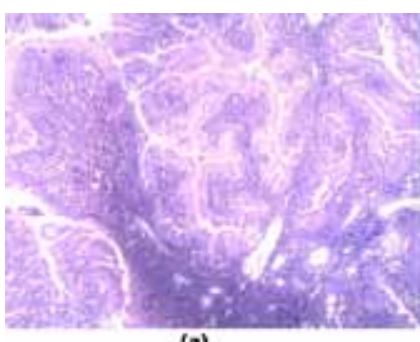

(a)

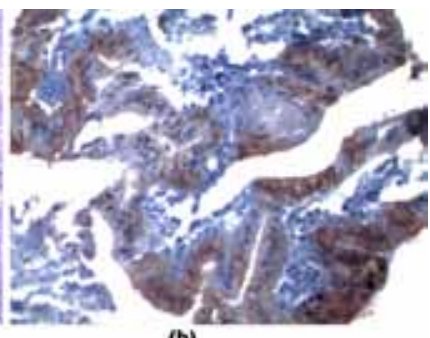

(b)
Fig.3: Sections of palatal PLGA showing (a) Histopathological (haematoxylin \& eosin staining), (b) Immunohistochemistry positivity photomicrographs.

incisional biopsy is taken but multiple small incisional samples are required for large tumors [4]. Some studies advocate histopathological evaluation of fully excised tumor and clinicopathological correlation necessary for final diagnosis [7].

In view of small size of the fumor in our patient and FNAC suggestive of a benign lesion, an excisional biopsy with safety margins was performed. The histopathologicaland immunohistochemistry examinations were suggestive of PLGA. Immunohistochemistry is an important tool in dealing with salivary gland neoplasms. PLGA cells are positive for Vimentin, CK 7 and S100 negative for glial fibrillary acid protein (GFAP). PA shows immunoreactivity to GFAP and none to Vimentin [3].

For PLGA standardized care is surgical resection. Adjuvant radiotherapy is only recommended in cases with inadequate margins, perineural or perivascular spread and/or lymph node involvement [5]. The overall prognosis for PLGA is favorable with a disease specific survival rate of $97.6 \%$ on an average follow up of 9.6 years [8]. However, in some studies recurrence rate as high as $33 \%$ is seen and distant metastasis and even transformation to higher grade adenocarcinoma has been noted suggesting the need for lifelong monitoring including chest $X$-ray $[2,4]$. PLGA should be included in the differential diagnosis of any fixed, firm, painless 
palatal mass with intact overlying mucosa.

\section{Conclusion}

A probing work up including adequate sized biopsy and immunohistochemistry is required to diagnose PLGA. PLGA should be considered in the differential diagnosis of a minor salivary gland tumor and particularly the characteristic cytomorphologic and immunohistochemistry features of PLGA should be kept in mind so as to differentiate it from PA and ACC.

\section{References}

1. Luna MA, Wenig BM. Polymorphous low grade adenocarcinoma. In: Barnes L, Eveson JW, Reichart P, Sidransky D (eds). World health organization classification of tumours. Pathology and genetics of head and neck tumours. $1^{\text {st }}$ edition. Lyon: IARC Press; 2005:223-224.

2. Seethala RR, Johnson JT, Barnes EL, Myers EN. Polymorphous low-grade adenocarcinoma. The University of Pittsburgh Experience. Arch Otolaryngol Head Neck Surg. 2010;136:385392.

3. Pintor MF, Figuera L, Martinez B. Polymorphous low grade adenocarcinoma: Review and case report. Med Oral Patol Oral Cir Buccal. 2007; 1 2(8):549-551

4. Evans $H$, Luna M. Polymorphous low grade adenocarcinoma of minor salivary glands. A study of 40 cases with long term follow up and evaluation of the importance of papillary areas. Am J Surg Pathol. 2000;24(10):13191328.

5. Hunter JB, Smith RV, Gensler MB. Low Grade Papillary Adenocarcinoma of the palate: the significance of distinguishing it from Polymorphous Low Grade Adenocarcinoma. Head and Neck Pathol. 2008;2:316-323.

6. Fatahzadeh M. Palatal polymorphous low grade adenocarcinoma: case report and review of diagnostic challenges. Arch Orofac Sci. 2012;7(2):92-100.

7. Abu El-Naaj I, Leiser Y, Wolff A, Peled $M$. Polymorphous low grade adenocarcinoma: case series and review of surgical management. J Oral Maxillofac Surg. 69;7:1967-1972.

8. Castle JT, Thompson LDR, Frommelt RA, Wenig $B M$, Kessler HP. Polymorphous low grade adenocarcinoma: a clinicopathologic study of 164 cases. Cancer.1999;86(2):207-219. 